University of Nebraska - Lincoln

DigitalCommons@University of Nebraska - Lincoln

Faculty Publications, Department of Child, Youth, and Family Studies

Child, Youth, and Family Studies, Department of

2021

SensiTray: An Integrated Measuring Device for Monitoring

Children's Mealtime Dietary Intake

Hunter S. Guru

Anthony D. Weng

Santosh Pitla

Dipti Dev

Follow this and additional works at: https://digitalcommons.unl.edu/famconfacpub

Part of the Biomedical Commons, Biomedical Devices and Instrumentation Commons, Developmental Psychology Commons, Family, Life Course, and Society Commons, Other Psychology Commons, and the Other Sociology Commons

This Article is brought to you for free and open access by the Child, Youth, and Family Studies, Department of at DigitalCommons@University of Nebraska - Lincoln. It has been accepted for inclusion in Faculty Publications, Department of Child, Youth, and Family Studies by an authorized administrator of DigitalCommons@University of Nebraska - Lincoln. 


\title{
SensiTray: An Integrated Measuring Device for Monitoring Children's Mealtime Dietary Intake
}

\author{
Hunter S. Guru, ${ }^{1}$ Anthony D. Weng, ${ }^{2}$ \\ Santosh Pitla, ${ }^{3} \&$ Dipti A. Dev ${ }^{4}$ \\ 1 Computer Science, Stanford University, Stanford, CA USA; hguru@stanford. \\ edu \\ 2 Economics, Stanford University, Stanford, CA USA; ad2weng@stanford.edu \\ 3 Biological Systems Engineering, University of Nebraska - Lincoln, Lincoln, NE \\ USA; spitla2@unl.edu \\ 4 Child, Youth \& Family Studies, University of Nebraska - Lincoln, Lincoln, NE \\ USA; ddev2@unl.edu
}

\begin{abstract}
Childhood nutrition establishes consumption norms that affect an individual's health over the course of their lives. However, early nutrition interventions to establish such norms are uncommon owing to the various inefficiencies associated with current methods of measuring childhood nutrition. Here, we present an loT measuring device, called the SensiTray, which accurately tracks mealtime intake in a child-friendly and cost-effective fashion. Principal technologies underlying the SensiTray (including mass-sensing technologies, microcontrollers) are identified and analyzed, along with other design choices. Operation of the SensiTray is explained with special attention given to SensiTray software peripherals and algorithms. Preliminary testing consisted of static and dynamic simulations (i.e. food weights constant and changing), with the SensiTray demonstrating a high degree of accuracy in both formats. Future
\end{abstract}

Published in 2021 IEEE International Instrumentation and Measurement Technology Conference (I2MTC), 17-20 May 2021.

DOI: $10.1109 /$ I2MTC50364.2021.9459897

Copyright (C) 2021 IEEE. Used by permission.

This paper was peer-reviewed at the direction of IEEE Instrumentation and Measurement Society to the acceptance and publication. 
development of the SensiTray may focus on design iteration, additional testing, and experimentation with various mealtime data visualizations to precipitate positive dietary changes.

Index terms - childhood obesity, obesity, nutrition, smart plate, smart tray, loT

\section{Introduction}

Though childhood nutrition is critical to normal development and establishing healthy consumption norms, in the U.S., the diet quality of children from 2 to 5 years of age often fails to meet age-specific recommendations. Many young children have lower than recommended intake of whole grains, "superfoods" like legumes and beans, and crucial fruits and vegetables; instead, their diets are supplemented by high consumption of refined grains and other foods rich in sodium, sugar, and empty calories [1]. Early establishment of poor diet quality increases the risk of childhood obesity and associated chronic conditions including diabetes and cardiovascular diseases [2]. Consequently, in both research and practice, there exists a necessity to measure nutritional intake from mealtime food consumption.

\section{A. Existing Methods}

Given the lack of a commercially feasible and accurate method to evaluate children's dietary intake, nutrition intervention evaluations are often either not performed or performed inaccurately; consequently, the establishment of poor nutrition norms is often undetected during this most critical time period. The current standard for measuring children's dietary intake is the manual food waste method; each individual food item served to a child during a meal is manually weighed before and after consumption. The difference in food item is then used to compute a projection of the child's nutritional intake for that meal. However, the manual food waste method is infeasible to employ on a large scale in childcare settings due to an assemblage of factors [3] including, but not limited to, high demand for trained meal observers, time and labor necessary for observer training, and high level of intrusiveness interrupting natural consumption behavior. 
Children's food consumption may also be assessed through digital photos, but this method is cost and resource intensive, with limited feasibility in childcare settings [4]. Furthermore, childcare directors have raised concerns about the usage of cameras or cell phones in childcare settings, even if their sole purpose is to take multiple pictures of a child's food tray during a mealtime. Specifically, the digital photo method requires that a picture be taken of the child's tray: before the child starts eating, every time the child takes additional servings of a food item served, and at the end of the meal [5].

\section{Proposed Method}

We introduce an loT measuring device, capable of measuring mealtime food intake, referred to as the SensiTray. The SensiTray is especially designed for children, and it is presented as an alternative to the standard school lunch tray. The tray operates by measuring timeseries data of up to five food items and utilizing the USDA nutrition database to approximate accurate nutritional information [6]. Additionally, we developed scalable software peripherals to allow appropriate stakeholders (childcare administrators, parents, and researchers) to view, control, and visualize tray data.

As shown in [Fig. 1], the tray has a bamboo enclosure, about the size of a standard school lunch tray. Within the enclosure, there is an acrylic housing which encases the electronics; this includes a set of load cells to measure the weight of food items, an SD card to store data collected (in the case of a wireless network limitation), and an ESP32 micro-controller. Above the electronics is a silicone mat to provide a softer, food-grade surface to consume food. Unlike a standard school lunch tray, the tray contains no grooves or indents for food rather, magnetic dishware "snaps" into the tray. Keeping costs of the tray to a minimum was a focus of ours, and assembling it costs approximately $\$ 30$ (USD).

\section{A. Mass Measurement}

Measuring nutrition information accurately and precisely reduces to a problem of choosing a reliable and accurate mass sensor. To keep 

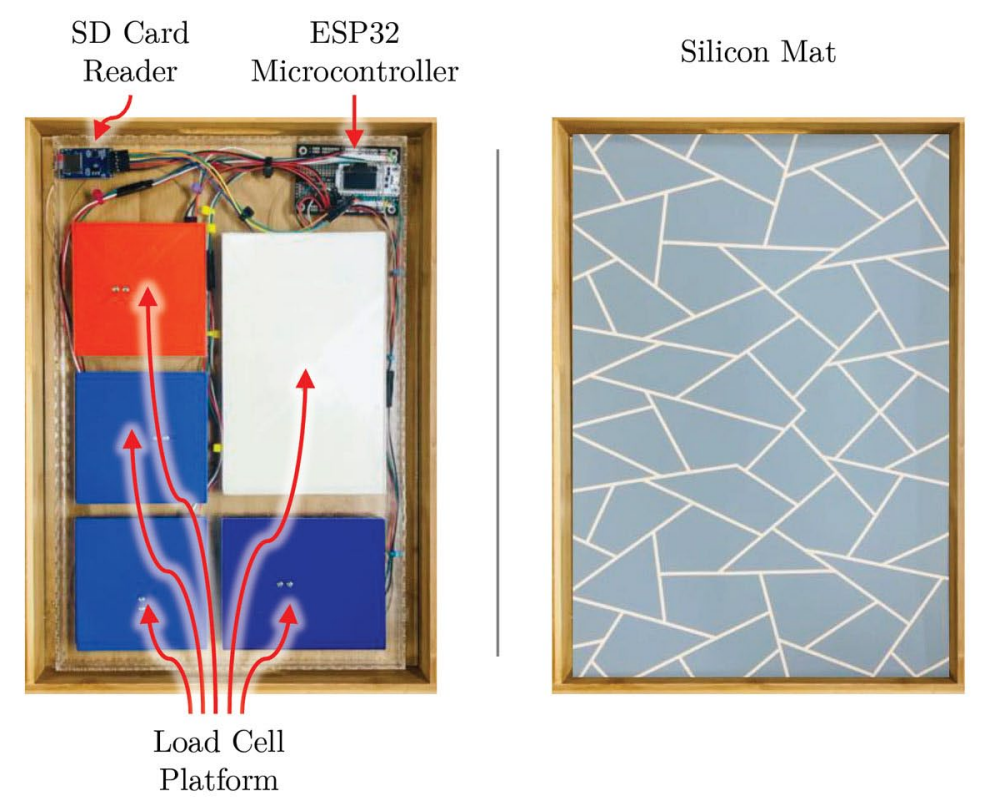

Magnetic Dishware

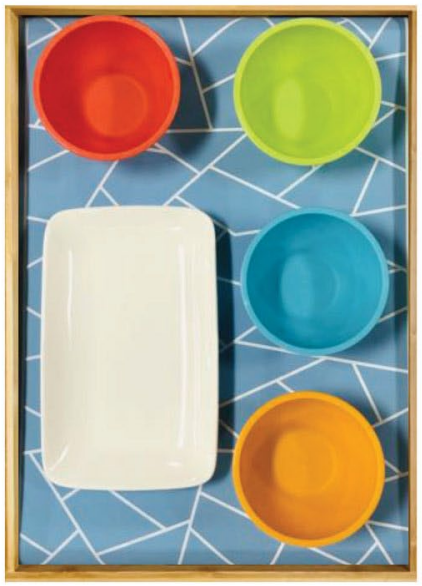

Fig. 1. The three layers of the tray (electronics, protective silicone, dishware).

the tray thin, it is necessary for the sensor to have a low profile. Forcesensing capacitors (FSCs) were the initial choice of sensor for the tray, for their virtually imperceptible thickness (high-end FSCs were selected for testing purposes) and supposed advantages in both repeatability and sensitivity over force-sensing resistors [7]. However, throughout extensive testing, FSCs were inaccurate for distinguishing between fine-grained levels of pressure. Error was on order of $\Theta(m \log$ $t$ ) upon testing, with $m=$ mass, $t=$ time. Price of the sensors was also prohibitively expensive.

Load cells were identified as a viable alternative. The sensors are force transducers that convert external, applied forces into a measurable electrical signal. They come in variety of sizes and operable ranges; typically, the sensors are beams made to deform to a certain degree within their operable range.

A mass-detecting base [Fig. 2] was designed to "sandwich" load cells. Plastic spacers were 3D printed to add separation between the faces of the load cells. To magnify smaller changes in mass, load cell amplifiers were connected. In initial experimentation, load cells had a high level of accuracy and failed at a lower rate. They were also more durable, and required far less "repeated" calibration. 


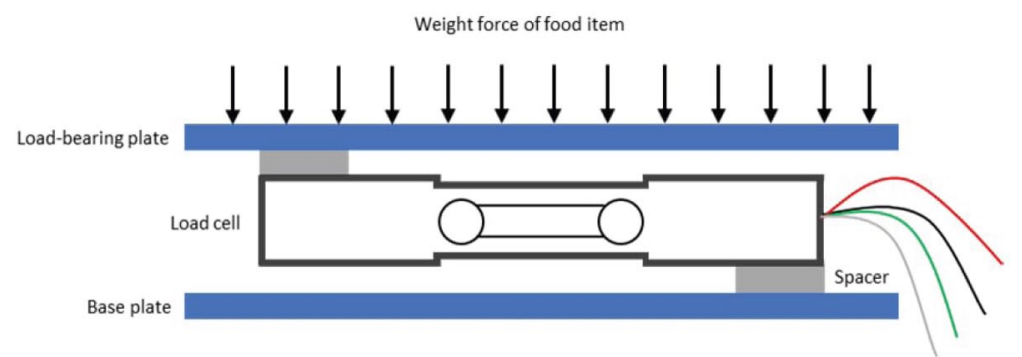

Fig. 2. Load cell "sandwiched" by two spacers, a base, and a platform. Adapted from [8]

Sensor calibration was conducted in a two-step process. 100 raw readings were obtained and averaged from each sensor with zero load. This averaged value was each sensor's "offset." Then, a reference mass was placed on each sensor, and 100 raw readings were obtained and averaged again. This value, minus the sensor's initial offset, was then divided by the known mass to obtain each sensor's scaling factor. As indicated in [Fig. 3], the relationship between raw signal count and reference mass was nearly perfectly linear. Additional tests to identify

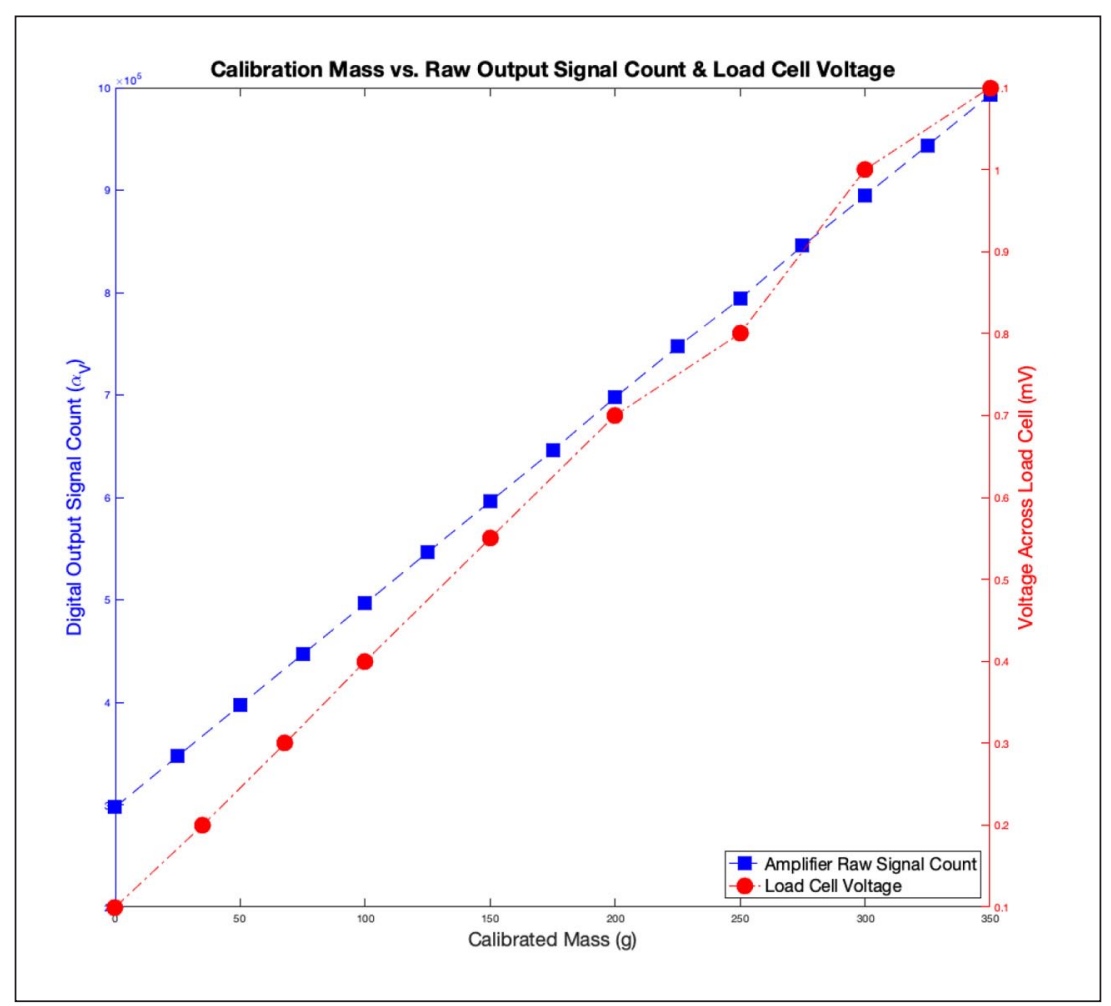

Fig. 3. Measuring voltage across load cell (circled) and raw digital signal from amplifier (squared) as a response to varied reference masses. 
drift were also completed; masses were left on load cell sensors for 72 hours, and no real drift-based error was observed. For most food items, error detected in testing was $\Theta(1)$ (constant factor) within the reasonable bounds of food weight $(<1 \mathrm{~kg})$. Further analysis of results is discussed in Section IV.

\section{B. Other Electronics}

The SensiTray utilized an integrated ESP32 microcontroller to connect to load cells. The ESP32 can communicate wirelessly via internet and Bluetooth. This board is commonly used in loT projects due to low price and energy consumption (powered by a rechargeable battery, not shown in [Fig. 1]).

The amplifier receives an analog voltage signal proportional to the mass applied to the load cell, which is forwarded to a programmable gain amplifier, via an input multiplexer. The signal is then converted to a digital value through the onboard 24-bit analog-to-digital converter. The ESP32 receives the serial data through $I^{2} \mathrm{C}$ communication protocol. Upon reading force data from each of the load cells, the ESP32 writes the data to JSON. The JSON packet is sent to an AWS server at a constant interval (30 seconds). If the on-board WiFi module is unable

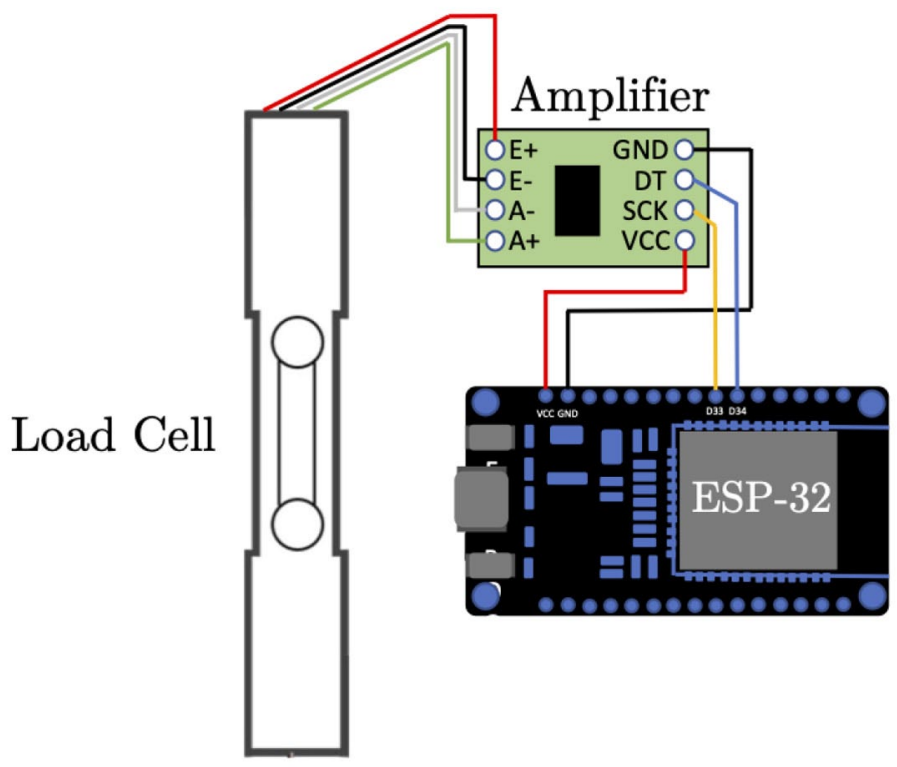

Fig. 4. Each Load cell requires four GPIO pins for power, ground, data transfer, and clock. The ESP32 contains 30 GPIO ports. 
to connect to a network, the data is stored on an SD card and is automatically uploaded the next time it connects. The tray broadcasts messages via the MQTT protocol, and an AWS server is subscribed to these JSON messages. The server organizes the streams of JSON packets collected during a meal into an associated meal entry; this data can be accessed by external APIs for client applications or exported for use by other researchers.

\section{Creating a Surface}

As seen in [Fig. 1], a series of five disjoint load cell platforms are contained in the SensiTray. The platforms must somehow be "unified", with intent to create a food-grade, dishwasher friendly surface. This design choice also prevents severe damage from occurring in the case of spillage. While it may seem like an opaque plastic platform may be adhered to the top of the tray, this gives rise to the issue of cross-sensitivity. Since force is distributed throughout the entirety of a unified surface, food items on the edges of a platform potentially interfere with the readings of adjacent platforms. This effect is inescapable, although it is negligible for a material with low rigidity. Silicone was an easily available choice, which met all of these criteria; upon reiterating all accuracy tests with and without the surface, a t-test indicated very little disparity between collected data.

Due to the presence of a surface, it becomes difficult to ensure that a SensiTray user places a dish or a food item directly on one of the platforms. Thus, it was beneficial to add strong neodymium magnets to each of the platforms, thereby establishing "hot regions" which attract dishes with magnetic surfaces. Similar testing was conducted to ensure magnets would not interfere with tray accuracy.

\section{Using The Tray}

\section{A. First Time Setup}

The first time a user sets up a SensiTray, they download the SensiTray mobile application built on Flutter - a Dart-based mobile application framework. After connecting to the tray via Bluetooth, the user 
is given the opportunity to configure WiFi credentials, give the tray a "nickname", and assign a parental figure who can access measurements from the tray. Nowhere in the registration process is a child's name captured, allowing the data to be completely anonymized by child.

\section{B. Populating Meal Data}

At this time, the SensiTray does not automatically detect food items using image recognition; hence, prior to using the tray, one first must acknowledge which food items are being served. To identify food items, childcare administrators can create menus of the food items they plan to serve on a specific day at a particular meal time (breakfast, lunch, snack). These menus are stored in a secure database where they may later reviewed or reused as needed.

In our experience, color coordinating food vessels was an effective means of ensuring that the correct load cells were measuring the correct food items; when uploading menu items, software automatically assigns a particular color to each food item, signifying which bowl certain food items should be served in. Nutritional information for these food items are automatically pulled from USDA's FoodData Central, which provides nutrition facts per $100 \mathrm{~g}$.

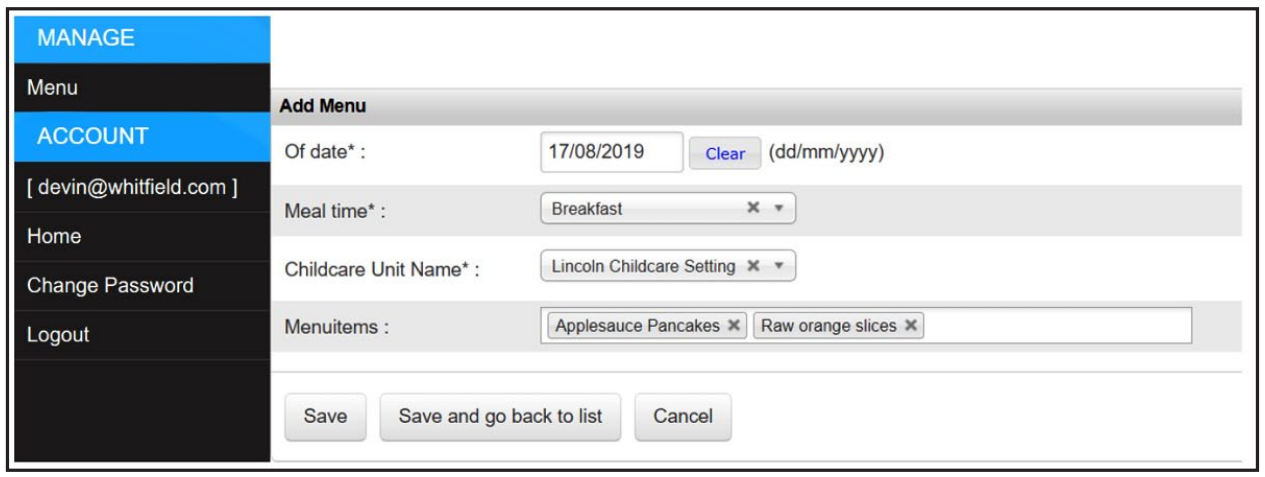

Fig. 5. An administrator specifies a breakfast menu with food items. 


\section{Consuming Food}

After placing each dish on its respective platform - without any food items - turning on the SensiTray automatically tares all dishware and establishes connection with an AWS server. When the tray chimes, food can be served or consumed. As food is consumed, intermittent pressure applied to a platform (potentially by a spoon or a fork) may cause spikes and noise in data. Operating under the assumption that these instances regularly occur less than half the time, a modified median filter [9] is applied to filter such noise. Consider a stream of load cell data $\{f[n]\}$, which records $k$ readings per second. Define a window of size $W=2 t_{e} \cdot k$, where $t_{e}$ denotes the average time to consume a morsel of food; then, the output of the median filter, $\{g[n]\}$ is defined as

$$
g[i]:=\operatorname{med}\left(\left\{f\left[i-t_{e} k\right], \ldots, f[i], \ldots, f\left[i+t_{e} k\right]\right\}\right)
$$

By assumption, less than half the window contains spiked values, hence, the median of such a window should ignore the spike. Given a meal of length $t_{m^{\prime}} k t_{m}-W$ data points are collected, with total computation involving $O\left(W\left(k t_{m}-W\right)\right)=O\left(k^{2} t_{e}\left(t_{m}-t_{e}\right)\right)$ in resources.

If the meal consists of only one serving, total food consumed, or $C$, can be modeled as $C:=g[n-1]-g[0]$, where $n$ is the total number of discrete values collected from the stream. Measuring data as a stream also allows for consumption to be modeled in the event of multiple servings, with

$$
C:=\sum_{i=0}^{n-2} \frac{|g[i+1]-g[i]|-(g[i+1]-g[i])}{2}
$$

Intuitively, this function sums up all of the incremental pairs $(i, i+1)$ such that $g[i+1]-g[i]<0$. Although intermediary points demonstrated increased average error during noisy interactions with the tray, an estimate of the eating trajectory is vastly improved from the filter [Fig. 6]. 


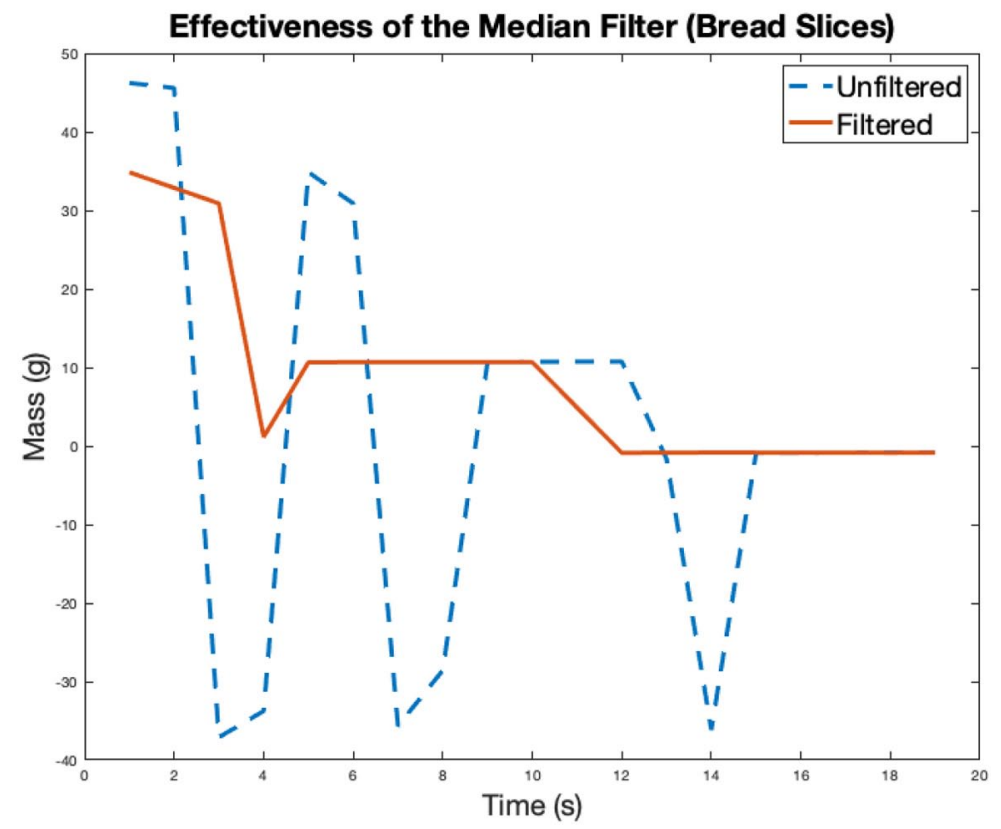

Fig. 6. A median filter drastically diminishes the effect of noise from repeatedly removing slices of bread and returning them to the tray.

\section{Results}

\section{A. Static Analysis}

Initially, SensiTray's ability to measure masses on all platforms in a static setting needed to be tested. The tray was tested with precision weights and food items. Masses of food items were estimated by an external scale. A precision (.1 mg resolution) digital analytical lab balance was chosen as the reference scale, assessed by the manufacturer to have linearity of $\pm 3 \mathrm{mg}$ and repeatability of $\pm 2 \mathrm{mg}$, with a maximum permissible error well within the minimal important difference.

For tests with the reference masses ranging from $0-100 \mathrm{~g}$, a platform on the tray was randomly chosen, and in half of the trials, a $100 \mathrm{~g}$ mass was placed in the adjacent platform to measure effects of crosssensitivity. A mass estimate was given as the mean of $n=10$ independent collected measures from the load cell. Errors relative to the reference masses [Fig. 7] were heteroskedastic, and the maximum observed standard deviation was $.7030 \mathrm{~g}$. Hence, an upper bound on 


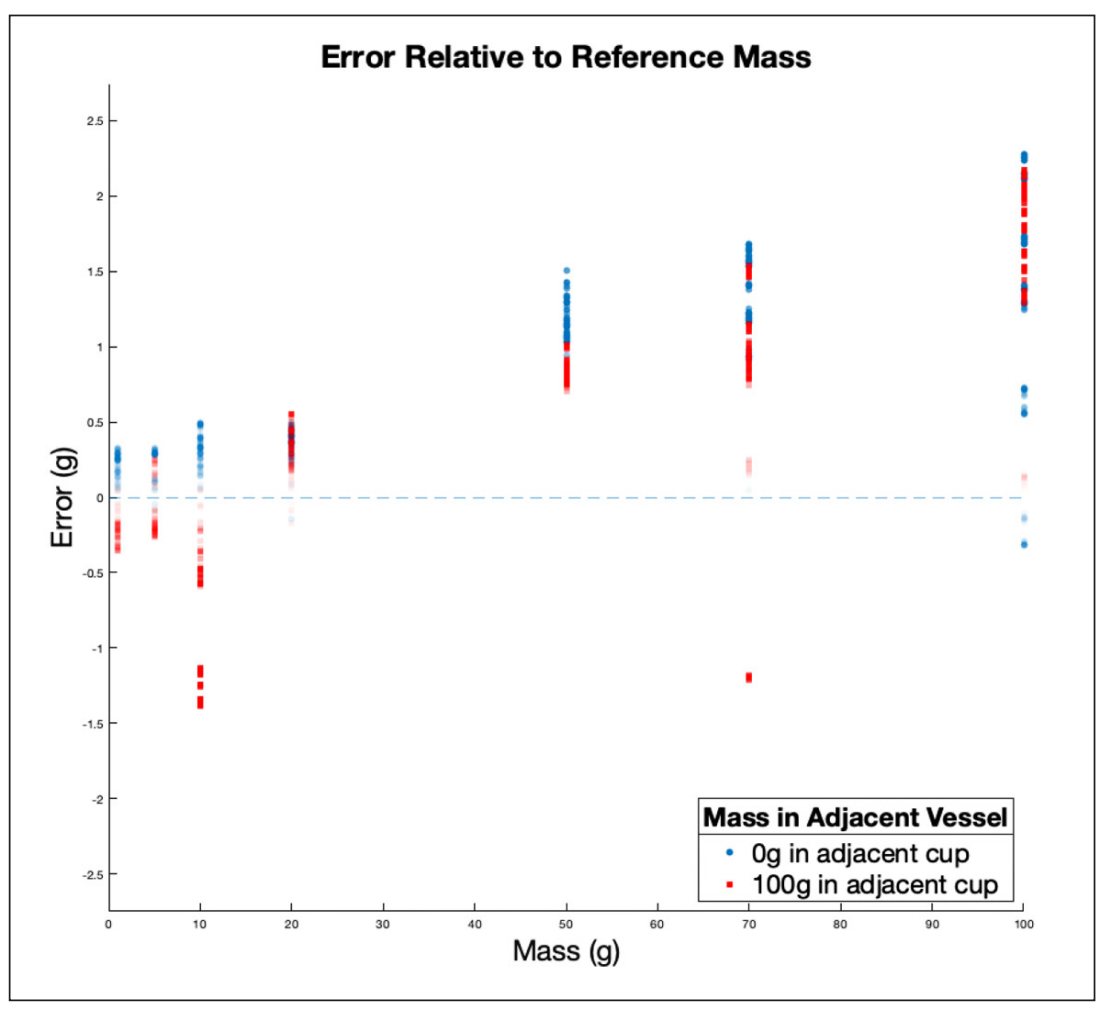

Fig. 7. Distribution of errors, relative to reference mass, with and without mass $(100 \mathrm{~g})$ placed on adjacent platform.

standard error was estimated as $\approx 0.22 \mathrm{~g}$. Relative uncertainties were collected, and fell beneath .13 for all mass measures.

Foods were chosen with an intent to maximize the diversity of shape, consistency, and weight distribution. The items tested included canned mangoes, apple sauce, carrots, milk, graham crackers, rice, and beans.

All food items were individually placed in each of the vessels, to test for cross sensitivity. This was repeated ten times, and data collected is indicated in Table 1 (mass measurements were rounded to the nearest gram). From our testing, we note that the maximum average error is limited to \pm 3 grams, and the average error across all food items was just \pm 1.29 grams. Unsurprisingly, the accuracy of a food item's mass measurement does not seem to be linked to the food group said item belongs to. 
Table I Static Mass Accuracy Testing

\begin{tabular}{lrrr} 
Food Item (Food Group) & True Mass $(g)$ & Avg. Measured (g) & Error $( \pm g)$ \\
\hline Canned mangoes (Fruit) & 113 & 114 & +1 \\
Apple Sauce (Fruit) & 250 & 249 & -1 \\
Baby carrots (Veg.) & 157 & 154 & -3 \\
Milk (Dairy) & 201 & 202 & +1 \\
Graham cracker (Grain) & 120 & 122 & +2 \\
Rice (Grain) & 84 & 84 & +0 \\
Beans (Protein) & 56 & 55 & -1 \\
\hline
\end{tabular}

\section{B. Dynamic Analysis}

Rather than simply measuring the final value of the tray, Eq. (1) was utilized as a measure of consumption. Testing occurred with both one and multiple servings. Eating was simulated by removing morsels of food by silverware (peaches, apple sauce, oats) and hand (graham crackers, doughnut); these morsels were moved to individual cups for future comparison [Fig. 9]. The error was nearly identical for all food items, aside from doughnuts, which were contained on platform D [Fig. 8]. At $t=23$, the sensor measured more than the "true" consumption and after correcting at $t=24$ it appeared as if servings had been added to the platter. When lifting the doughnut to "take a bite", the readings were lower than the true amount consumed. This amounted to a near $14 \%$ error. At this time, the median filter needs improvement for items which involve removing and returning said items for longer periods of time.

Similarly, when multiple (three) servings were introduced, for food items besides doughnuts, no significant changes are observed - the error in doughnuts was only amplified (on average 19\% across three tests). Therefore, these tests indicate that error for food items was related to $t_{e}$ and $t_{m}$; food items with higher spread in $t_{e}$ experienced more error. Adjusting the filter's window size, coupled with adjusting the rank of the filter from $r=n / 2$, may help remediate these issues.

Analysis was conducted to measure the similarity and reliability between dynamic measures amongst ten time series, where an initial amount of food with mass $M_{i}$ was distributed across the vessels, 


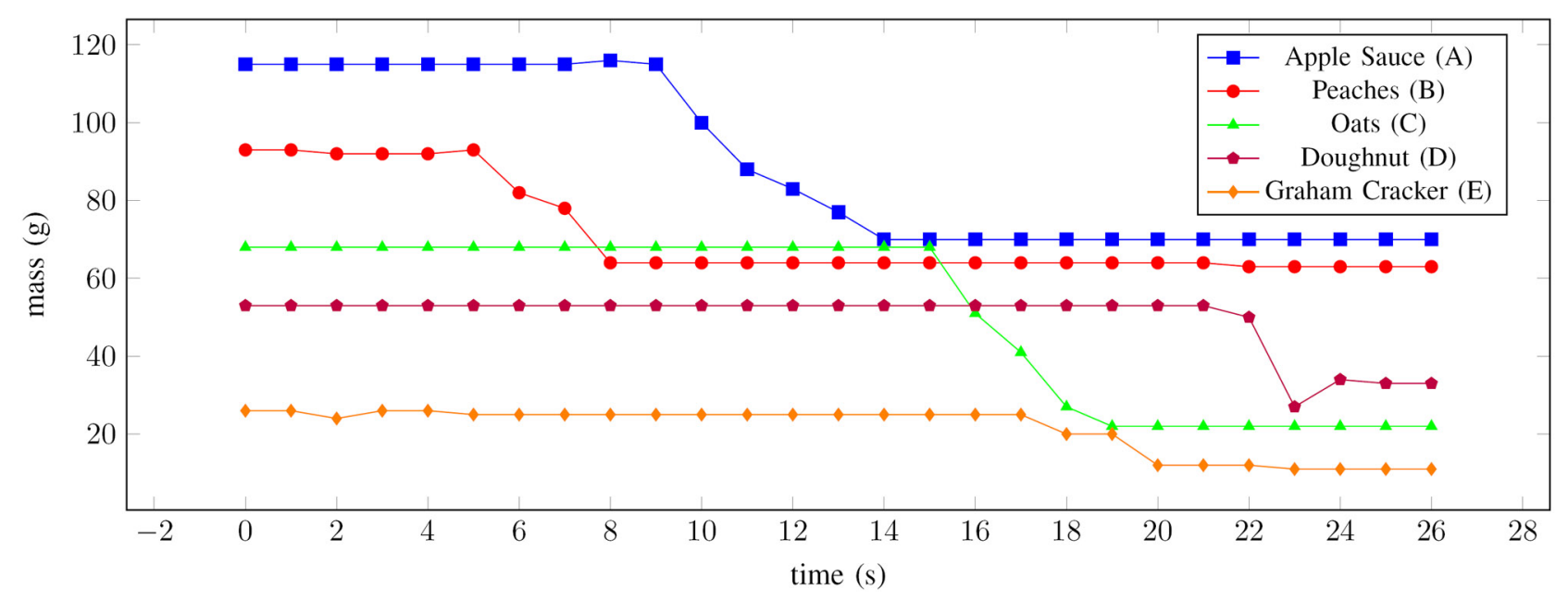

Fig. 8. Time series received during 30 second data capture, without increasing servings.

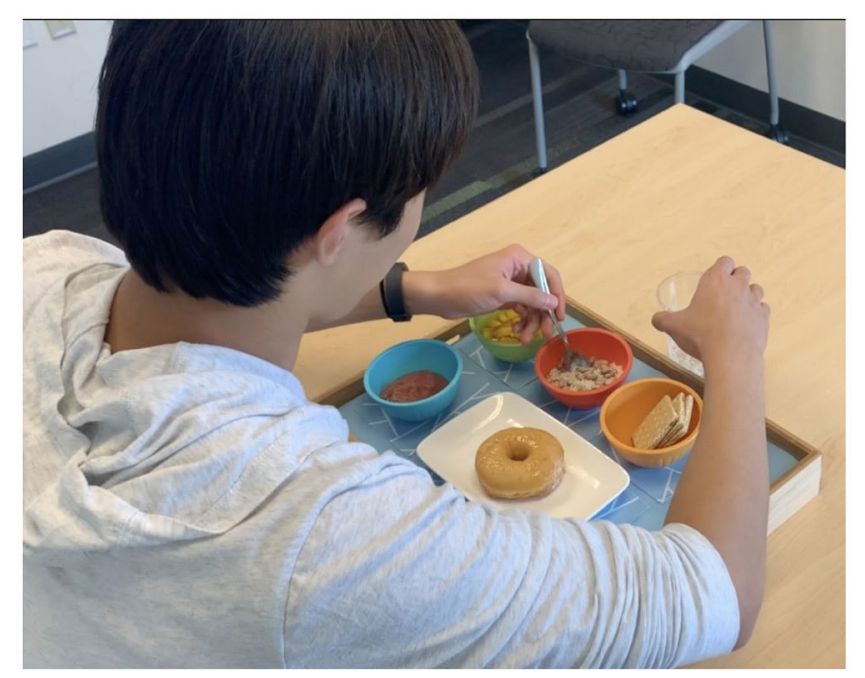

Fig. 9. Oats are scooped from a vessel into an external container.

and similarly sized morsels of various food items were removed periodically across $t$ discrete time intervals. Dynamic time warping was utilized to align time series and calculate pairwise unnormalized distance, $\Delta(x, y)$. A standardized similarity measure over $[0,1], S(x, y)$, was defined to compare strength of similarity,

$$
S(x, y):=1-\frac{\Delta(x, y)}{M_{i} t}
$$




\begin{tabular}{|c|c|c|c|c|c|c|c|c|c|c|}
\hline 2 & 3 & 4 & 5 & 6 & 7 & 8 & 9 & 10 & External & \\
\hline \multirow[t]{10}{*}{.9936} & .9916 & .9882 & .9901 & .9958 & .9921 & .9930 & .9862 & .9845 & .9939 & 1 \\
\hline & .9964 & .9916 & .9916 & .9931 & .9920 & .9950 & .9915 & .9900 & .9957 & 2 \\
\hline & & .9938 & .9931 & .9924 & .99927 & .9938 & .9944 & .9918 & .9960 & 3 \\
\hline & & & .9961 & .9901 & .9924 & .9901 & .9958 & .9900 & .9930 & 4 \\
\hline & & & & .9926 & .9950 & .9909 & .9945 & .9892 & .9936 & 5 \\
\hline & & & & & .9955 & .9945 & .9883 & .9865 & .9952 & 6 \\
\hline & & & & & & .9939 & .9909 & .9884 & .9950 & 7 \\
\hline & & & & & & & 9917 & .9908 & .9943 & 8 \\
\hline & & & & & & & & .9929 & .9909 & 9 \\
\hline & & & & & & & & & .9889 & 10 \\
\hline
\end{tabular}

Fig. 10. Pairwise similarity between food consumption time series, with an additional external scale utilized to measure mass for reference.

Similarity measures were extra-ordinarily high, with the smallest pairwise similarity exceeding .98 [Fig. 10].

\section{Brief Comparison to Other Existing Methods}

Existing methods to model nutritional intake over a period of time are mainly interested in measuring the difference between initial and final consumption [10]. Noninvasive recognition systems, such as software-aided photography classification systems, perform at approximately $80-85 \%$ mass classification accuracy for food items ranging between $70-80 \mathrm{~g}$ [11]. Our similar testing results found an approximate 95\% mass classification accuracy, with stronger, near constant uncertainty bounds. Additionally, the measurement methods presented allow potentially robust inter-meal consumption trajectory estimations, which may serve beneficial in research associated with eating patterns and meal habits.

\section{Future Work}

We see a multitude of directions to extend our findings. We are currently creating a thinner tray, in which we wish to include image recognition to avoid pre-meal information population. Improving the filter's consistency will also prove useful for diverse food items. Further testing (both short-term and long-term) with children as subjects is necessary to test the measurements in a highly unpredictable setting. 
We foresee the importance of assessing novel visualizations' ability to communicate data to precipitate positive dietary changes.

\section{Conclusion}

As an loT device which accurately collects time-series nutritional data in childcare settings, the SensiTray achieved major engineering aims, including:

- Design of a child-friendly, easily-operable, and cost-effective solution for accurate nutrition tracking,

- Integration with lightweight loT framework, for an autonomous system which may be more scalable than current manual food waste and digital photography methods,

- Development of software peripherals and algorithms, enabling stakeholders (children, parents, childcare providers) to plan, log, and visualize children's nutritional intake.

We are excited to further develop and test the SensiTray, with intentions to promote the device as an accurate, reliable instrument in conducting future childhood obesity studies.

Acknowledgment The authors would like to thank CheeTown Liew for helpful discussions and laboratory support. In addition, they thank Dr. Ashu Guru for software and database consultations.

\section{References}

[1] E. C. Banfield, Y. Liu, J. S. Davis, S. Chang, and A. C. Frazier-Wood, "Poor adherence to us dietary guidelines for children and adolescents in the national health and nutrition examination survey population," Journal of the Academy of Nutrition and Dietetics, vol. 116, no. 1, pp. 21-27, 2016.

[2] S. Xu and Y. Xue, "Pediatric obesity: Causes, symptoms, prevention and treatment," Experimental and therapeutic medicine, vol. 11, no. 1, pp. 15-20, 2016.

[3] C. Wolper, S. Heshka, and S. B. Heymsfield, "Measuring food intake: An overview." 1995. 
[4] D. A. Williamson, H. R. Allen, P. D. Martin, A. J. Alfonso, B. Gerald, and A. Hunt, "Comparison of digital photography to weighed and visual estimation of portion sizes," Journal of the American Dietetic Association, vol. 103, no. 9, pp. 1139-1145, 2003.

[5] E. L. Kenney, K. K. Davison, S. B. Austin, C. M. Giles, A. L. Cradock, R. M. Lee, and S. L. Gortmaker, "Validity and reliability of a simple, low-cost measure to quantify children's dietary intake in afterschool settings," Journal of the Academy of Nutrition and Dietetics, vol. 115, no. 3, pp. 426-432, 2015.

[6] [Online]. Available: https://fdc.nal.usda.gov/

[7] L. Martinelli, C. Hurschler, and D. Rosenbaum, "Comparison of capacitive versus resistive joint contact stress sensors," Clinical Orthopaedics and Related Research ${ }^{\circledR}$, vol. 447, pp. 214-220, 2006.

[8] S. Alves, "Troubleshooting load cell applications," May 2016. [Online]. Available: https://www.ee.co.za/article/troubleshooting-load-cell-applications. $\underline{\mathrm{html}}$

[9] G. Arce and M. McLoughlin, "Theoretical analysis of the max/median filter," IEEE transactions on acoustics, speech, and signal processing, vol. 35, no. 1, pp. 60-69, 1987.

[10] M. Archundia Herrera and C. B. Chan, "Narrative review of new methods for assessing food and energy intake," Nutrients, vol. 10, no. 8, p. 1064, 2018.

[11] W. Zhang, Q. Yu, B. Siddiquie, A. Divakaran, and H. Sawhney, "'snap-n-eat" food recognition and nutrition estimation on a smartphone," Journal of diabetes science and technology, vol. 9, no. 3, pp. 525-533, 2015. 